\title{
Leading for Well Being - Dignity as Cornerstone
}

\section{Michael Pirson ${ }^{1}$}

Published online: 22 July 2021

(C) The Author(s), under exclusive licence to Springer Nature Switzerland AG 2021

The Humanistic Management Journal was founded as a space for foundational research in a marginal and emerging domain of human organizing. The foundation of what we label humanistic is what famed biologist E.O. Wilson labeled the consilience of knowledge around who we are as human beings and what we want. He stated the basic existential questions of where we come from, who we are and where we are going have a novel vantage point that bridges many insights of the ancient wisdom traditions with those of evolutionary biology and even quantum physics.

Specifically evolutionary theory, the science of survival, points to insights that align with insights of religions and wisdom traditions: As a species we survive when we service the common good. This insight is a foundational challenge to traditional economics and management. As such we are finding ourselves in a space that has dominated human conversation and politics for ages. Human experience has been expressed as the pursuit of short term goals such as material wealth and personal success or alternatively as long term welfare and the contribution to the common good. The stories that we share as a human species remind us that the former is "bad" while the latter is "saintly". From the story of Moses and the ten commandments versus the worship of the golden calf, to the adulation of wealth versus the adulation of wisdom in Chinese and Greek culture to the current debates of shareholder value primacy and stakeholder well being: There is an underlying assumption of who we are as human beings and what we want. Success is often either equated with wealth or well-being. While this may be a trivial story to many, scholars and observers argue that this is the never-ending epic between good and bad, long-term and short term, other-oriented and self-oriented action, i.e. management.

As the Humanistic Management Journal we support sharing research that engages in that conversation while being scientific and evidence based. Much of economics and management research is, by contrast, assumption based- the main assumption being self- interest as exclusive motivator. Yet, given the ever solidifying consilience of knowledge there is much to be reimagined around how we research, teach and organize. In this issue we are publishing a number of articles that contribute to the science of human survival. Several papers specifically discuss the question of what we organize for - the 'telos'- the goal of human organizing.

Michael Pirson

pirson@fordham.edu 
Sandra Waddock's article on Well Being Economics Narratives for a Sustainable Future discusses many of the above mentioned challenges when shifting from short term to long term orientation. She argues that there is increasing interest in the notion of a wellbeing economy, and recognition that a coherent story or narrative is important in countering the strength of today's dominant economic narrative-neoliberalism. Yet there has been relatively little consensus on what such an idea might mean in practice, despite the proliferation of many different initiatives attempting to bring such an economy about. Many of these initiatives have allied with an aggregator called WEAll, the Wellbeing Economy Alliance. In an effort to determine what new economics/next economies initiatives mean by wellbeing economy, this study assesses the statements provided by WEAll allies to WEAll when seeking membership to see whether there is a consistent narrative about wellbeing economy that emerges. Four nuanced, yet related, versions of the wellbeing economy narrative emerged. "Transformation" is mainly a critique of business as usual, emphasizing transformation towards a wellbeing economy that critiques neoliberalism and emphasizes wellbeing of all people. The Nature-Centric or Planetary Boundaries perspective takes a more ecological perspective, emphasizing humanity's need to live and operate within planetary boundaries. The Good Life or People-Centric perspective offers a people-oriented narrative that emphasizes sustainability while ensuring that basic needs of all are met. The Integrated perspective is an integrated life-centered perspective that combines these interests and focuses on the wellbeing of both people and planet, recognizing the complexity and holistic nature of that task.

In a second article, Pamala J. Dillon explores the notion of Well Being through Dignity. In her article Virtuous Social Responsiveness: Flourishing with Dignity, Dillon argues that Corporate social responsibility (CSR) focuses organizational inquiry on the role of business in society and corporate social performance (CSP) provides a framework comprised of principles, processes and outcomes describing CSR performance. In contrast, virtuous social responsiveness (VSR) describes CSP from a humanistic management perspective, providing an alternative principle of social responsibility as the basis from which processes and outcomes flow. Incorporating humanistic management assumptions into the role of business in society, she argues, leads to social performance predicated on well-being creation and dignity promotion. VSR requires a principle of social responsibility grounded in eudaemonia, positioning well-being creation as the common good, as opposed to wealth creation. Dignity promotion within stakeholder relationships is a second requirement for VSR, indicated by altruist stakeholder culture and a collectivistic organizational identity orientation. Theoretical propositions are developed to describe a humanistic management perspective on CSP. A current example of an organization engaging in VSR is provided, illustrating potential CSP outcomes. A discussion of the importance of VSR is presented, along with implications and future research.

In a third contribution Paulina Roszkowska and Domenec Melé explore the interaction between Organizational Factors and its impact on Individual Ethical Behaviour. They develop the notion of an "Organizational Moral Structure." The authors argue that various organizational factors reported in the literature affect individual (mis)behaviour within a company. In their paper, the authors conduct a literature review thereof, and propose a notion of the "Organizational Moral Structure" defined as a comprehensive framework of interrelated organizational factors that condition, incite or influence good or bad moral behaviour of individuals within the organization. Drawing from a wide bibliographical review and their own reflection on recent business scandals, the authors identify seven constituents of the “Organizational Moral Structure": 1) leader's values and character, 2) vision and exercise of 
power, 3) corporate control systems, 4) internal network of influence, 5) organizational culture, 6) internal and competitive pressures, and 7) external influences. The "Organizational Moral Structure" is proposed as a reflective framework for humanistic management and as an invitation to further research in this field. The authors provide recommendations on how a manager oriented towards humanistic management can use the OMS to secure and promote well-being and dignity of company's employees.

As a specific example of organizational practices that aim to protect dignity and enable human flourishing Chrystal Jaye, Lauralie Richard, Claire Amos and Geoff Noller examine the managemet of sick leave in a university. In their article, "Managing Sick Leave in the University: Bureaucracy and Discretion" the authors examine the challenges for supervisors and managers of managing sick leave within a New Zealand university. The authors use a qualitative research design, interviewing 20 university staff across the academic and service divisions who had managerial roles. They apply Habermas' distinctions of technical instrumental, practical relational, and emancipatory critical transformative interests, and his twofold distinction of system and lifeworld to our analysis. The primary findings suggest that while the technical instrumental discourses were dominant within the university bureaucracy, managers (particularly front line managers) drew upon practical relational and emancipatory critical transformative discourses to justify the considerable discretion they exercised in managing sick leave. The authors find that far from being incidental, these humanistic elements are as much a part of the bureaucracy as the rational elements and are fundamental to the system's equilibrium.

Building on the notion of consilience Reetesh K. Singh and Mansi Babbar explore the notion of religious diversity at the workplace. The authors argue that globalization, increased migration, and mobility of workforce necessitate the need to study religious diversity in organizations, which has not yet received adequate academic attention of management scholars. Their paper attempts to define and understand the nuances of religious diversity with the help of certain theories from psychology and sociology domains. It aims to present the legal provisions of different countries regarding workplace religious discrimination and endeavours to synthesize and analyze the pros and cons of religious diversity at workplace. The paper follows a systematic literature review approach. The descriptive analysis of search results and thematic analysis of the literature reviewed has been presented. It is found that the theories from other domains can be borrowed to understand the root cause of different perceptions for religious diversity. The favourable and unfavourable outcomes of workplace religious diversity have been identified and categorized at two levels i.e. individual and organizational. It is realized that the favourable outcomes of bringing religion to workplace outnumber the unfavourable outcomes and therefore it is time to leverage workplace religious diversity. Lastly, the paper attempts to equip the human resource managers with certain strategies to better deal with religious diversity issues at the workplace.

In a further contribution Vanessa Schäffner is exploring the challenge of Artificial intelligence for human flourisihing in the area of self-driving cars. In her paper" Between Real World and Thought Experiment: Framing Moral Decision-Making in Self-Driving Car Dilemmas", Schäffner asks how driverless vehicles should respond to situations of unavoidable personal harm? Her paper takes up the case of self-driving cars as a prominent example of algorithmic moral decision-making, an emergent type of morality that is evolving at a high pace in a digitized business world. As its main contribution, it juxtaposes dilemma decision situations relating to ethical crash algorithms for autonomous cars to two edge cases: the case of manually driven cars facing reallife, mundane accidents, on the one hand, and the 
dilemmatic situation in theoretically constructed trolley cases, on the other. The paper identifies analogies and disanalogies between the three cases with regard to decision makers, decision design, and decision outcomes. The findings are discussed from the angle of three perspectives: aspects where analogies could be found, those where the case of self-driving cars has turned out to lie in between both edge cases, and those where it entirely departs from either edge case. As a main result, the paper argues that manual driving as well as trolley cases are suitable points of reference for the issue of designing ethical crash algorithms only to a limited extent. Instead, a fundamental epistemic and conceptual divergence of dilemma decision situations in the context of self-driving cars and the used edge cases is substantiated. Finally, the areas of specific need for regulation on the road to introducing autonomous cars are pointed out and related thoughts are sketched through the lens of the humanistic paradigm.

In a final essay Elisa Maria Entschew is exploring one of the most prominent complaints of our time- the lack of time in an accelerated world of digital communication. She argues that digital communication between humans fundamentally changes the nature of communication. One inherent change is the acceleration of communication as a systematic change in societal life, particularly in the workplace. Often, the aim is to release time resources. However, the acceleration of communication also leads to the opposite: a lack of time. This paradoxical development can be based on an acceleration cycle whereby technologies seem to be a solution on the micro-level, but they are also a significant part of the problem on the meso-level.

The editorial team thanks all the authors and wishes all our readers a joyful read.

Publisher's Note Springer Nature remains neutral with regard to jurisdictional claims in published maps and institutional affiliations. 